\title{
The role of the microbiology laboratory in healthcare-associated infection prevention
}

\author{
Smilja Kalenić, Ana Budimir \\ Croatian Society for medical Microbiology and Parasitology, \\ Section for Healthcare-associated Infection Prevention and Control, Zagreb, Croatia
}

doi: 10.3396/ijic.V5i2.014.09

\section{Introduction}

Healthcare associated infections $(\mathrm{HAl})$ are a big problem worldwide, increasing morbidity and mortality of hospitalized patients, as well as increasing cost of healthcare. Many hospitals throughout the world have good systems for HAI prevention and control, but others have not and are forced to develop such systems nowadays. Having microbiology laboratory as a part of the hospital diagnostic laboratories, is a huge advantage for HAI prevention and control. Ideally, a microbiology laboratory should be set up inside the hospital, working every day on a 24 hour basis. If it is not possible, then hospital should contract microbiology services with the geographically nearest laboratory.

Microbiology laboratory has two main functions in the hospital: one is diagnosis of infection in individual patient, directly related to the patient care and the other is support to the HAI prevention and control. But the first role - diagnosis of infection - has also a function in HAI prevention: if the etiological diagnosis of infection is rapid and accurate, the patient will be treated properly at the beginning of infection, so time of dispersing microorganisms will be shorter.

Every microbiology laboratory should have quality assurance procedures in place, and if possible, external quality control of main procedures performed by the laboratory. Dramatic changes in microbiological diagnostic techniques in the last decades - rapid diagnostic methods and precise typing methods have emphasised even more the role of microbiology laboratory.

\section{Diagnosis of infections}

For the accurate etiological diagnosis of infection several conditions should be fulfilled: first, clinician should put right indication for this diagnostics; furthermore, the right sample should be taken; then, all data needed for the laboratory should be put on the request (name of the patient and the physician, location of the patient, date and time of specimen collection, working diagnosis or differential diagnosis, underlying patient conditions, antimicrobial chemotherapy if already instituted).

Specimens sent to the microbiology laboratory have to be taken on the right time (depending on the pathogenesis of the infection), from appropriate sites using proper techniques, in a quantity that will ensure good workout in the laboratory. Transport conditions from the patient to the laboratory are to be carefully maintained. Microbiology laboratory staff can assist in ensuring good specimens by educating clinical staff.

Rapid and accurate diagnosis and sensitivity test are of outmost importance for the direct patient care. In 
the laboratory methodology it is necessary to provide rapid tests (like pneumococcal urine antigen in the case of community acquired pneumonia, or rotavirus latex agglutination for detection of rotavirus in faeces etc). For diagnosis to be accurate, laboratory has to develop procedures for quality assurance and has external quality control system in function.

Microbiology laboratory should be able to examine blood, cerebrospinal fluid, urine, stool, wound exudate or swab, respiratory secretions, and perform basic serological tests (HIV, HBV, HCV, influenza).

Microbiological laboratory should be able to identify at least the following bacteria and fungi: Staphylococcus aureus, Escherichia coli, Group A streptococci, Group B streptococci, enterococci, Streptococcus pneumoniae, Haemophilus influenzae, Neiserria meningitidis and Neiserria gonorrhoeae, Salmonella, Shigella, Campylobacter jejuni/coli, Clostridium difficile, Pseudomonas aeruginosa, Klebsiella pneumoniae, other enterobacteria, Candida albicans, aspergilli (and other, depending on local epidemiological situation); also, it should be able to diagnose specific viral infections (eg some of viruses causing diarrhoeal diseases), but mostly using serological methods (HIV, $\mathrm{HBV}, \mathrm{HCV}$, influenza, or other viruses, depending on local epidemiological situation).
Methods used in the diagnosis of infections are:

1. Classical methods, like

- Direct smear

- Culture

- Antigen detection

- Serological tests

\section{Molecular methods (not very often used in routine work)}

- Hybridization

- Amplification

- Real-time amplification

As identification of the infectious agent is gradual, the laboratory has to communicate with the attendant clinician preliminary and definite diagnosis, as well as sensitivity test results (Figure 1).

For sensitivity testing the most used method is discdiffusion, although nowadays many laboratories are using automated methods. Some laboratories use MIC determination, either by classical MIC dilution techniques or by E-tests. Sensitivity test results are used immediately for the patient care. Adequate antimicrobial therapy leads to better outcomes and is vital for individual patient care, as well as the decrease of microorganism transmission to other patients. But also, laboratory should perform periodical reports of

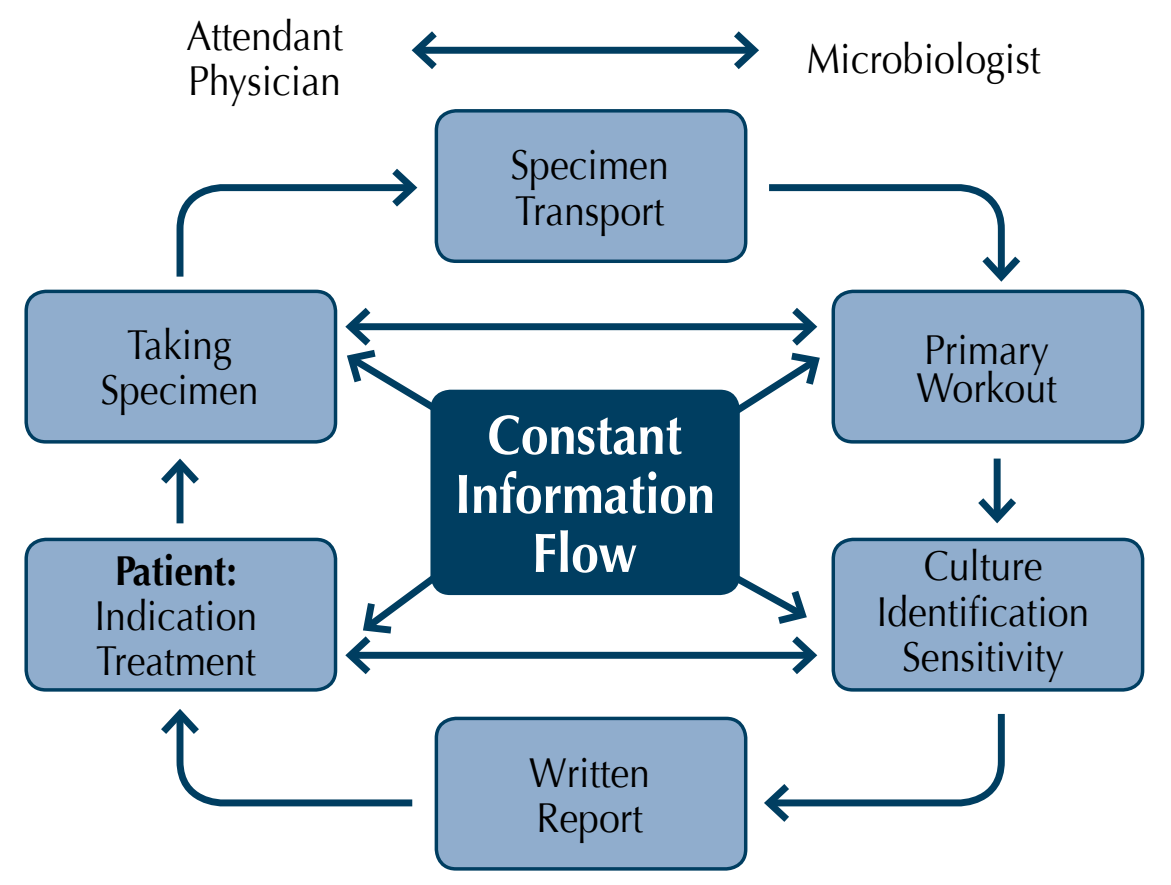

Figure 1. Communication between attendant physician and microbiologist for ethiological diagnosis of infection 
sensitivity of bacteria (broken down by bacteria and specimen) isolated in patient on the specific ward (unit) - and this would mostly be intensive care unit for the design of empiric therapy of infections in that specific ward (unit).

\section{The role in HAI prevention}

\section{Rapid communication}

Infection control practitioner's (ICP) day should ideally start in the microbiology laboratory, personally or virtually. This would be the best and the most rapid communication of everyday infectious problems ICP can encounter in the hospital. If this is not possible, then laboratory workers should communicate by phone or personally with the ICP every new important isolated pathogen in patients. This rapid communication is crucial and is the base of any good collaboration between microbiology laboratory and infection control.

\section{Accurate speciation and typing of isolated pathogen}

To be useful for epidemiological studies of HAI, isolated bacterial strains should be identified to the species level. Many times this is enough for definite epidemiological analysis of small clusters or even outbreaks of $\mathrm{HAI}$ if pathogenic bacteria are involved (e.g. Salmonella Enteritidis food poisoning outbreak originated from hospital kitchen). But, if the isolated bacteria are only opportunistic pathogens (e.g. S. aureus, E. coli, Enterobacter cloacae, enterococci etc) it is necessary to type those isolates to be sure that the cluster or outbreak patients are connected. To type means to determine characteristics which differ between different strains of the same bacterial species. Namely, the individual bacteria inside the same species could differ in their genome as much as $30 \%$, and in the individual bacterial clone (clone $=$ offsprings of the common ancestor) these differences are very small or even non-existent.

Typing of bacteria is possible by several methods. Main characteristics of typing methods are:

- Typability, i.e., the method types most or all strains of the same species

- Discriminatory power, i.e., the method differentiates between different types

- Inter/intra laboratory reproducibility, i.e., the method provides the same typing results in repeated testing on same or different sites
- The method has to be simple, unambiguous to interpret, and inexpensive

There are two groups of typing methods:

a) phenotyping

b) genotyping

a) Phenotyping means that we are determining characteristics that are expressed in the bacterial culture, like:

- Antigenic structure (serotyping)

- Physiologic properties/metabolic reactions (biotyping)

- Susceptibility to antimicrobial agents (resistotyping)

- Susceptibility to colicines (colicinotyping), or

- Susceptibility to bacteriophages (phage typing)

Very important limiting factor of phenotypic methods is the fact that bacterial genes are not always expressed and so two phenotypically different strains can actually have the same genetic background or two phenotypically identical strains can actually differ genetically. The other limiting factor is that sometimes one unique phenotype is widespread and frequent, and in that cases genotyping will be required for outbreak management.

b) Genotyping can demonstrate the relatedness or difference between two isolates of the same species very precisely. Diagnosis can be rapid - because it is not dependent on waiting for bacterial growth in cultures. Genotyping requires sophisticated and expensive equipment and materials and also well trained staff. Genotyping methods are either imaging (based on restriction of part or whole genome with the endonucleases and electrophoresis of fragments), or sequence-based (which means definite biological information and is more and more used).

Some imaging methods have a low reproducibility, especially in interlaboratory comparisons, and interpretation of results is neither always simple nor unambiguous.

Genotyping methods are not very often used in routine microbiology laboratory. Therefore it is important for a hospital laboratory to be connected with research 
laboratories in the country, or other types of central laboratories that are able to perform such genotyping in the time of the outbreak.

\section{Participation in outbreak management}

The early isolation of a new or unusual microorganism enables the ICP to take appropriate measures to stop it from spreading at the very beginning. Any new isolates should be reported immediately to the wards and the ICP. Laboratory staff may also report clustering of infections (two related isolates in different patients in the same time frame), which then enables ICP to go and check these cases.

In an outbreak suspected to be caused by one and the same causative agent it is very helpful (sometimes even necessary) for the agent to be isolated, identified to the species level and also typed.

Bacterial typing determines if two or more strains are really related and if they differ from strains that are not epidemiologically connected. If two (or more) strains are unrelated, the patients do not belong to the same outbreak, and it is very simple to interpret that pattern. But, if two (or more) strains are related after we performed strain typing it is very difficult to say that the patients are involved in an outbreak without sound epidemiological analysis.
Sometimes during the outbreak analysis, epidemiologist would ask laboratory some additional types of tests, to clarify the situation. These can be for example microbiological tests of blood products, environmental surfaces, disinfectants and antiseptics, air, water, hands of personnel, nostrils of personnel.

\section{Participation in HAI surveillance}

Surveillance of HAI is an important task of a well organized infection control. If outcome surveillance is performed, inherent to some infection definitions (BSI, UTI) is an isolation of the causative organism, but also important in all other infection definitions. Furthermore, in alert or multi-drug resistant (MDR) organisms surveillance the role of microbiology laboratory is crucial. The most important 'alert' microorganisms are:

- Methicillin-resistant Staphylococcus aureus (MRSA)

- Vancomycin-intermediate $S$. aureus (VISA)

- Vancomycin-resistant enterococci (VRE)

- MDR Pseudomonas aeruginosa

- MDR Acinetobacter baumannii

- MDR Mycobacterium tuberculosis

- ESBL enterobacteria

- Clostridium difficile

MRSA infections in surgical intensive care unit

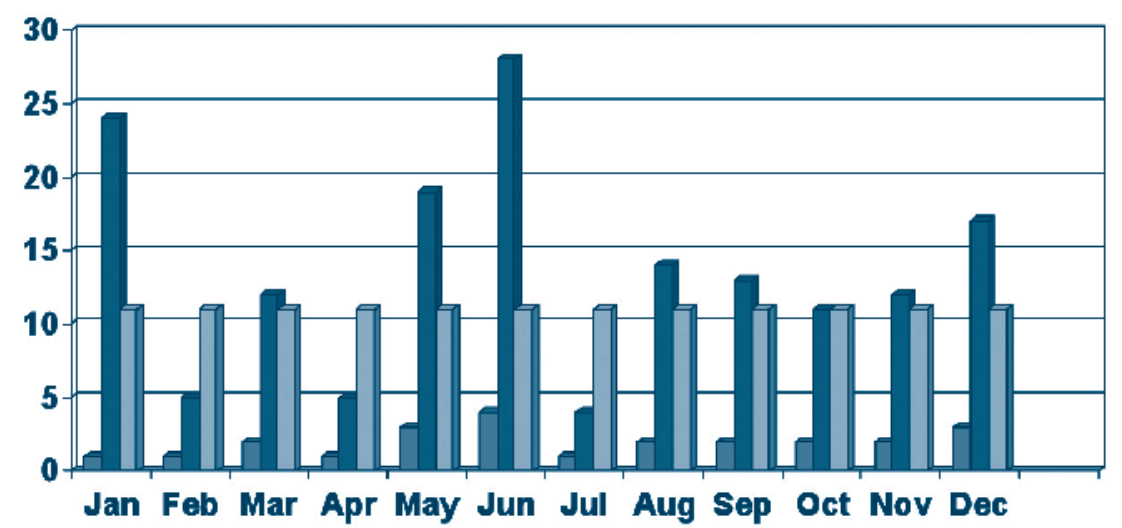

$\square$ No of infected pts

inf/1000 pt days $\square$ mean inf/1000 pt days

Figure 2. MRSA infections in Surgical Intensive Care Unit, time series 
The microbiology laboratory should produce routine reports to allow the ICP to make graphs for specific pathogens, wards, and groups of patients. A 'baseline incidence' can be then established and any new isolate incidence can be compared with this incidence. If such graphs are in the form of time series, they will enable the ICP to discover the beginning of an outbreak earlier than it would be possible clinically (Figure 2).

Periodic reports also demonstrate trends of specific pathogens and can be very useful in planning preventive measures. Microbiology laboratory also participates in routine process surveillance monitoring sterilization, monitoring dialysis fluid. Sometimes it may be useful to routinely monitor some other processes, like infant formula and instrument disinfection.

\section{Participation in resistance surveillance}

The surveillance of bacterial resistance to antimicrobial agents is very important task of microbiological laboratory. It serves to an antibiotic management team in hospitals, to make policies for antibiotic use with the ultimate goal to decrease this resistance. Resistance reports are usually periodical (yearly reports) and it is very useful to have resistance surveillance organised on regional or country or even international level, to cope with the resistance development on a broader scale (for example European Antimicrobial Resistance Surveillance System).

\section{Education of HAI prevention personnel and other healthcare workers}

Microbiologist acts also as an educator for infection control personnel, first about characteristics of microorganisms that are important for epidemiology of infections, then about normal flora, difference between contamination, colonisation and infection with specific organisms, furthermore about interpretation of microbiological reports and charts.

Microbiology laboratory staff serves also as educators to all other healthcare workers, about specimen taking and transport and also interpretation of microbiology findings. Microbiology laboratory serves as education point for basic clinical microbiology for medical and nursing students.

\section{Microbiologist as a member of Infection Control Committee (ICC)/Infection Control Team (ICT) and Antibiotic Committee (AC)}

As microbiologist has specialist knowledge necessary for infection control and antibiotic use he/she is an indispensable member of ICC/ICT and AC in hospitals. Other members of ICC and AC in hospital do not have the knowledge of microbiology. It is not surprising then, that in US almost half of the ICC chairs are laboratory personnel.

It is also important that the microbiologist is a medical doctor, because he/she will thus understand pathogenesis of infection as well as pathogenesis of HAl, not only characteristics of an organisms, will be able to understand pharmacokynetics and pharmacodynamics of antimicrobial agents - and will be able to communicate with clinical and nursing colleagues on the same level of understanding disease, therapy and epidemiology.

Only exceptionally, if there is no medical doctor available, a properly educated scientist could be microbiologist too, but in that situation he/she has to be very closely connected with infectious disease specialist.

\section{Conclusion}

Microbiology laboratory is becoming an integral part of HAI prevention programmes. The emergence of new pathogens, and new resistances in old pathogens, makes microbiology laboratory indispensable for successful prevention of HAI, not only outbreaks, but sporadic cases too.

Different typing methods, available on site or in the central laboratory of the country, are necessary for outbreak management. As microbiology and laboratory methods are developing very fast, there is a sound need for a specialist microbiologist to be a part of the infection control team, infection control committee and antibiotic committee. Only very close communication, collaboration and cooperation between microbiology laboratory and infection control personnel can fulfil the duties of successful infection prevention and control in healthcare institutions. 


\section{Bibliography}

- Diekema DJ, Pfaller MA. Infection Control Epidemiology and Microbiology Laboratory. In: Murray PR, Editor in Chief. Manual of Clinical Microbiology, $8^{\text {th }}$ edn. ASM Press, Washington, DC 2003; 129-138.

- Stratton CW IV, Greene JN. Role of the Microbiology Laboratory in Hospital Epidemiology and Infection Control. In: Mayhall CG, Ed. Hospital Epidemiology and Infection Control, $3^{\text {rd }}$ edn. Lippincott, Williams \& Wilkins, Philadelphia, 2004;1809-1825.

- Poutanen SM, Tompkins LS. Molecular Methods in Nosocomial Epidemiology. In: Wenzel RP, Ed. Prevention and Control of Nosocomial Infections, $4^{\text {th }}$ edn. Lippincott, Williams \& Wilkins, Philadelphia, 2003; 481-499.

- Gill VJ, Fedorko DP, Witebsky FG. The clinician and the Microbiology Laboratory. In: Mandell GL, Bennett JE, Dolin R, Eds. Mandell, Douglas and Bennett's Principles and Practice of Infetious Diseases, $6^{\text {th }}$ edn. Elsevier, Philadelphia, 2005; 203-241.
- Peterson LR, Hamilton JD, Baron EJ, et al. Role of clinical microbiology laboratory in the management and control of infectious diseases and the delivery of health care. Clin Infect Dis 2001; 32: 605-611.

- Jarvis WR. The Role of the laboratory in Control of healthcareAssociated Infections. In: Jarvis WR, Ed. Bennett \& Brachman's Hospital Infections, $5^{\text {th }}$ Edn. Lippincott, Williams \& Wilkins, 2007:121-150.

- Van Eldere J. Changing needs, opportunities and constraints for the $21^{\text {st }}$ century microbiology laboratory. Clin Microbiol Infection 2005; 11 (Suppl 1): 15-18. 